\title{
Comparison of early and long-term follow-up results of percutaneous mitral balloon valvuloplasty and mitral valve replacement
}

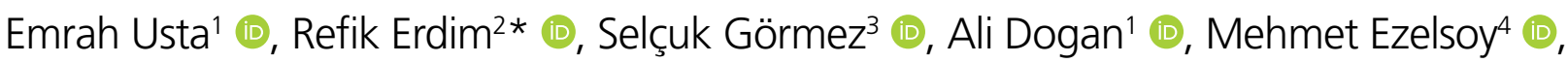 \\ Serkan Kahraman ${ }^{1} \mathbb{D}$, Muhammed Bayram ${ }^{4}$ (D), Nuran Yazicioğlu ${ }^{5}$
}

\begin{abstract}
SUMMARY
BACKGROUND: Percutaneous mitral balloon valvuloplasty and mitral valve replacement have been the treatment options for mitral stenosis for several years, however, studies that compare these two modalities are very rare in the literature.

Objectlve: In this article, we aim to investigate the comparison of clinical results of percutaneous mitral balloon valvuloplasty and mitral valve replacement. Methods: 527 patients with rheumatic mitral stenosis, treated with percutaneous mitral balloon valvuloplasty or mitral valve replacement (276 patients with percutaneous mitral balloon valvuloplasty and 251 patients with mitral valve replacement) from 1991 to 2012 were evaluated. The demographic characteristics, clinical, echocardiographic and catheterization data of patients were evaluated retrospectively. The results of early and late clinical follow-up of patients after percutaneous mitral balloon valvuloplasty and mitral valve replacement were also evaluated. Results: The mean follow-up time of the percutaneous mitral balloon valvuloplasty group was 4.7 years and, for the mitral valve replacement-group, it was 5.45 years. The hospital stay of the percutaneous mitral balloon valvuloplasty group was shorter than that of the mitral valve replacement group ( 2.02 days vs 10.62 days, $\mathrm{p}<0.001$ ). The hospital mortality rate of percutaneous mitral balloon valvuloplasty and mitral valve replacement were $0 \%$ and $2 \%$ respectively $(p=0.024)$. In the percutaneous mitral balloon valvuloplasty group, early postprocedural success rate was $92.1 \%$. The event-free survival of percutaneous mitral balloon valvuloplasty and mitral valve replacement was found to be similar. While reintervention was higher in percutaneous mitral balloon valvuloplasty-group $(p<0.001)$, mortality rate was higher in mitral valve replacement-group $(p<0.001)$.

Concluslon: Percutaneous mitral balloon valvuloplasty seems to be more advantageous than mitral valve replacement due to low mortality rates, easy application of the procedure and no need for general anesthesia.

KEYWORDS: Mitral Valve. Balloon Valvuloplasty. Outcome, Treatment. Follow-Up Studies.
\end{abstract}

\section{INTRODUCTION}

The most common cause of mitral stenosis is rheumatic involvement. Acute Rheumatic Fever (ARF) is a multisystem, autoimmune disease caused by group-A beta-hemolytic streptococcal infections. It is still a serious health problem in many developing societies, and one of the leading causes of morbidity and mortality ${ }^{1-3}$.
Carditis is the most important sequela of ARF that causes morbidity and mortality. It is observed in 40-60\% of patients with rheumatic fever ${ }^{4}$. Carditis typically reveals itself as valvulitis. Mitral valve is the most frequent involvement site, while aortic and tricuspid valves are less frequently involved. Isolated mitral stenosis occurs in $25 \%$ of rheumatic valvular

\footnotetext{
IIstanbul Bilim University, Faculty of Medicine, Department of Cardiology - Istanbul, Turkey.

${ }^{2}$ Acıbadem Mehmet Ali Aydınlar University, Kadıkoy Hospital, Clinic of Cardiology - Istanbul, Turkey.

${ }^{3}$ Acıbadem Mehmet Ali Aydınlar University, Faculty of Medicine, Department of Cardiology - Istanbul, Turkey.

${ }^{4}$ Istanbul Bilim University, Faculty of Medicine, Department of Cardiovascular Surgery - Istanbul, Turkey.

${ }^{5}$ Florence Nightingale Şişli Hospital - Istanbul, Turkey.

*Corresponding author: errefik@hotmail.com

Conflicts of interest: the authors declare there is no conflicts of interest. Funding: none.

Received on August 15t, 2020. Accepted on August 24, 2020.
} 
heart diseases, while mitral stenosis and mitral regurgitation exist concurrently in $40 \%$ of the patients. The primary symptoms of mitral stenosis are fatigue and exercise intolerance. Dyspnea in normal daily physical activities, orthopnea and pulmonary edema may occur as the disease progresses. Mitral valve area in normal healthy adults is $4-6 \mathrm{~cm}^{2}$. If it is below $2 \mathrm{~cm}^{2}$, that is called mild mitral stenosis. A valve area between 1 to $1.5 \mathrm{~cm}^{2}$ is assessed as moderate stenosis, while one below $1 \mathrm{~cm}^{2}$ is severe mitral stenosis ${ }^{5}$. Echocardiography is the most widely used and most useful method in the diagnosis and monitoring of mitral stenosis.

The treatment of mitral stenosis consists of three modalities; medical treatment, percutaneous mitral balloon valvuloplasty (PMBV) and surgery, particularly mitral valve replacement (MVR).

The early therapeutic approach for patients with severe mitral stenosis was only surgical. With the advent of technology, percutaneous commissurotomy techniques have developed significantly ${ }^{6}$. In 1982, Dr. Kanji Inoue described the Inoue technique, using a novel single balloon device for percutaneous mitral commissurotomy ${ }^{7}$. At first, PMBV was described as an alternative treatment option to surgical mitral commissurotomy for mitral stenosis ${ }^{6}$. Clinical trials comparing the long-term results of PMBV and surgery demonstrated that both modalities produce similar hemodynamic improvement ${ }^{7}$. Today, PMBV is accepted as the preferred treatment in selected patients due to shorter hospital stay, lack of general anesthesia and lower morbidity rates than any other surgical techniques? ${ }^{7}$. MVR is performed for mitral insufficiency and patients with calcified mitral valve or left atrial thrombus.

We aimed to compare the echocardiographic parameters and the result of early and late clinical follow-up of patients after PMBV and MVR.

\section{METHODS}

The study consisted of 527 patients with rheumatic mitral valve stenosis that were treated with PMBV or MVR (276 patients with PMBV and 251 patients with MVR) in our tertiary clinic between January 1991 and December 2012. All patients were followed up for at least 6 months with echocardiography and 12 months with clinical evaluations. The patients who undergone concomitant coronary artery bypass grafting, aortic valve replacement, tricuspid valve procedures or ablation procedures for atrial fibrillation were excluded. The treatment strategy for patients with mitral stenosis (MS), PMBV or MVR was determined by the attending cardiologist or cardiovascular surgeon and was based on echocardiographic and clinical findings. The demographic characteristics, clinical, echocardiographic and catheterization data of patients were evaluated retrospectively. Pretreatment clinical (functional capacity, medical history, concomitant disease), echocardiographic parameters and long-term follow-up of the patients were obtained.

The main end points included in hospital early follow-up and long-term follow-up were defined as death, need of early surgery, need of reintervention, pericardial tamponade, embolic stroke and serious hemorrhage. Early surgery was defined as need of MVR or re-MVR as a complication of the procedure at hospitalization. Need of reintervention was defined as re-PMBV and re-MVR on long-term follow-up. Serious hemorrhage was defined as bleeding leading to decrease in hemoglobin level of $5 \mathrm{~g} / \mathrm{dL}$ or to the need of surgery for bleeding control (without dental/nasal/skin/hemorrhoids), as well as intravenous vasoactive agents.

\section{Echocardiographic evaluation}

2-dimensional and color Doppler echocardiographic evaluation was performed in all patients before and after the procedure. In addition to routine measurements, the mitral valve area was calculated by the planimetry of mitral valve orifice in parasternal short axis view and continuous wave Doppler technique was used to calculate the mitral gradient and the peak pressure gradient of tricuspid regurgitation for estimated systolic pulmonary arterial pressure (sPAP).

\section{Statistical analysis}

In this study, statistical analysis was made by SPSS (Statistical Package for Social Sciences) version 16.0. $\chi^{2}$, Fisher's exact $\chi^{2}$ test were used for comparison of descriptive statistical methods (mean, standard deviation), as well as categorical variables, incidence and rate. Student's t-test was used for the comparison of variable mean of the two groups in parametric assumptions. Mann-Whitney U test was used in nonparametric assumptions. Wilcoxon signed-rank and marginal homogeneity tests also were used when needed. $\mathrm{p}<0.05$ was considered for statistical significance.

\section{RESULTS}

In our study, PMBV group's mean age was $40.88 \pm 11.56$ and MVR group's mean age was $51.49 \pm 11.51(\mathrm{p}<0.05)$. In the PMBV group, $84.8 \%$ of patients were women; in the MVR group, $68.9 \%$ were women $(\mathrm{p}<0.05)$. Pre-procedure left atrium diameter was $5.08 \pm 0.68 \mathrm{~cm}$ in the PMBV group and $5,49 \pm 0.87 \mathrm{~cm}$ in the MVR group, which was statistically significant $(\mathrm{p}<0.05)$. In the PMBV group, pre-procedure rhythm was sinus in $71.4 \%$ of patients, whereas in the MVR group, pre-procedure rhythm was sinus in $41.4 \%$ patients $(\mathrm{p}<0.05)$. 
Baseline clinical and echocardiographic characteristics for all patients are summarized in Table 1. After the intervention, the hospital stay of the PMBV group was significantly shorter than that of the MVR (2.02 \pm 1.75 days; $10.62 \pm 4.53$ days, $\mathrm{p}<0.001)$. The mean follow-up time of the PMBV group was 4.7 years, and, for the MVR group, it was 5.45 years $(\mathrm{p}=0.053)$.

The hospital mortality rates of PMBV and MVR were $0 \%$ and $2 \%$, respectively. In the PMBV group, early postprocedural success rate was $92.1 \%$, whereas $7.9 \%$ of patients needed surgical intervention during hospital stay. Comparison of early results of the PMBV and MVR groups are summarized in Table 2.

The mortality rates of the PMBV group and the MVR group were $0 \%$ and $4 \%$, respectively $(\mathrm{p}<0.001)$. The reintervention rate of the PMBV group was $16.3 \%$, and this rate was $2.4 \%$ for the MVR group ( $\mathrm{p}<0.001)$. The rate of eventfree survival of PMBV after the follow-up was $81 \%$ and, in the MVR group, it was $88 \%(\mathrm{p}=0.107)$. The comparison of long-term follow-up results of the PMBV and MVR groups is summarized in Table 3.

Table 1. Baseline clinical and echocardiographic characteristics.

\begin{tabular}{|c|c|c|c|c|}
\hline & PMBV $(n=276)$ & $\operatorname{MVR}(n=251)$ & \multirow{2}{*}{$t$} & \multirow{2}{*}{$\mathrm{p}$} \\
\hline & mean \pm std & mean \pm std & & \\
\hline Age & $40.88 \pm 11.56$ & $51.49 \pm 11.51$ & -10.54 & $0.001 * *$ \\
\hline Pre-procedural MVA $\left(\mathrm{cm}^{2}\right)$ & $1.00 \pm 0.22$ & $1.04 \pm 0.21$ & -2.178 & $0.029 *$ \\
\hline Pre-procedural mean gradient $(\mathrm{mmHg})$ & $13.68 \pm 5.55$ & $13.00 \pm 4.10$ & -0.474 & 0.633 \\
\hline Pre-procedural LA diameter $(\mathrm{cm})$ & $5.08 \pm 0.68$ & $5.49 \pm 0.87$ & -6.23 & $0.001 * *$ \\
\hline \multirow[t]{2}{*}{ Pre-procedural sPAP (mmHg) } & $51.87 \pm 14.01$ & $53.02 \pm 13.23$ & -1.383 & 0.167 \\
\hline & $\mathrm{n}(\%)$ & $\mathrm{n}(\%)$ & $\chi^{2}$ & $p$ \\
\hline Sex (female) & 84.8 & 68.9 & 17.908 & $0.001 * *$ \\
\hline Pre-procedural rhythm SR & 71.4 & 41.4 & \multirow{2}{*}{1.867} & \multirow{2}{*}{$0.001 * *$} \\
\hline AF & 33.3 & 58.6 & & \\
\hline Previous procedure & 11.6 & 23.1 & 0.010 & $0.001 * *$ \\
\hline Pre-procedural MR None & 51.8 & 23.1 & \multirow{5}{*}{63.608} & \multirow{5}{*}{$0.001 * *$} \\
\hline+1 & 37.7 & 51.2 & & \\
\hline+2 & 8.0 & 21.5 & & \\
\hline+3 & 2.6 & 12.0 & & \\
\hline+4 & 0.0 & 0.0 & & \\
\hline Pre-procedural FC NYHA 1-2 & 46 & 50.2 & \multirow{2}{*}{0.035} & \multirow{2}{*}{0.850} \\
\hline NYHA 3-4 & 54 & 49.8 & & \\
\hline
\end{tabular}

${ }^{*} \mathrm{P}<0.05$; PMBV: percutaneous mitral balloon valvuloplasty; MVR: mitral valve replacement; MVA: mitral valve area; LA: left atrial; sPAP: systolic pulmonary arterial pressure; SR: sinus rhythm; AF: atrial fibrillation; MR: mitral regurgitation; FC: functional class; NYHA: New York Heart Association.

Table 2. Comparison of early results of percutaneous mitral balloon valvuloplasty and mitral valve replacement groups.

\begin{tabular}{|c|c|c|c|c|c|}
\hline & & $\begin{array}{c}\text { PMBV }(n=276) \\
\text { mean } \pm \text { std }\end{array}$ & $\begin{array}{c}\text { MVR }(n=251) \\
\text { mean } \pm \text { std }\end{array}$ & z & $p$ \\
\hline \multicolumn{2}{|l|}{ Hospital Stay (day) } & $2.02 \pm 1.75(2)$ & $10.62 \pm 4.53(10)$ & -19.40 & $0.001 *$ \\
\hline & & $n(\%)$ & $\mathrm{n}(\%)$ & $\chi^{2}$ & $p$ \\
\hline \multirow{2}{*}{ Death } & No & $276(100)$ & $246(98)$ & \multirow{2}{*}{5.551} & \multirow{2}{*}{$0.024^{*}$} \\
\hline & Yes & $0(0)$ & $5(2)$ & & \\
\hline \multirow{2}{*}{ Urgent Surgery } & No & 257 (93.1) & $251(100)$ & \multirow{2}{*}{15.99} & \multirow{2}{*}{$0.001^{* x}$} \\
\hline & Yes & $19(6.9)$ & $0(0)$ & & \\
\hline
\end{tabular}

"p<0.05; PMBV: percutaneous mitral balloon valvuloplasty; MVR: mitral valve replacement. 


\section{DISCUSSION}

In our retrospective clinical study, we demonstrated that the hospital mortality rate of PMBV was significantly lower than MVR. The event-free survival of PMBV and MVR was found to be similar. The need of reintervention was higher in the PMBV group than in the MVR group, which was statistically significant, however, the mortality rate of MVR was higher than PMBV's.

Almost all of the rheumatic mitral stenosis patients need intervention ${ }^{7}$. In the patients with inappropriate anatomic and clinical conditions for percutaneous approach, MVR and other surgical procedures can be performed. There are several studies that compare PMBV and other surgical procedures, such as open or closed mitral commissurotomy. Nevertheless, there are very few studies that compare early and long-term complications of PMBV and MVR. Although success rates of these modalities have been proven, complications can be seen in follow-up duration.

Early randomized, controlled studies showed similar clinical outcomes for both PMBV and MVR ${ }^{8,9}$. The limitation of previous clinical studies was the heterogeneity for both patient groups; moreover, open mitral commissurotomy was included in both studies. In our study in the PMBV group, the mean age was 40.88 , and $84.8 \%$ of patients were women, which are both statistically significant factors in comparison to the MVR group. In the PMBV group, pre-procedure rhythm was sinus rhythm in $71.4 \%$ of patients, whereas, in the MVR group, pre-procedure rhythm was atrial fibrillation in $58.6 \%$ of patients. These baseline characteristics were similar to previous studies 9

Immediate procedural success for both of these modalities has been shown in some randomized clinical trials $s^{8-14}$. Early procedural success for PMBV is defined as MVA $>1.5 \mathrm{~cm}^{2}$ without significant mitral regurgitation (MR) ${ }^{8-9,11}$. In the PMBV group, early postprocedural success rate was $92.1 \%$ in our study, which was relatively higher when compared to previous studies.

MR is the most common complication of PMBV. According to previous studies, severe MR after PMBV was observed in between $7.5-18.5 \%{ }^{15-18}$ of patients. In our study, severe MR occurred in 3.6\% of patients, which was relatively low compared to other studies. Mild to moderate MR is one of the most important determinant factors to patient selection for PMBV or MVR. Due to the aforementioned reason, the MVR

Table 3. Comparison of long-term follow-up results of the percutaneous mitral balloon valvuloplasty and mitral valve replacement groups.

\begin{tabular}{|c|c|c|c|c|c|}
\hline & & $\begin{array}{c}\text { PMBV }(n=276) \\
\text { mean } \pm \text { std }\end{array}$ & $\begin{array}{c}\text { MVR }(n=251) \\
\text { mean } \pm \text { std }\end{array}$ & z & $\mathrm{p}$ \\
\hline \multicolumn{2}{|l|}{ Clinical follow-up (year) } & $4.70 \pm 4.16(3)$ & $5.45 \pm 4.54(4)$ & -1.936 & 0.053 \\
\hline \multicolumn{2}{|l|}{ Echocardiographic follow-up (year) } & $3.52 \pm 3.01(2)$ & $4.93 \pm 4.64(3)$ & -0.397 & $0.001^{* \star}$ \\
\hline \multicolumn{2}{|l|}{ Postprocedural LA diameter (cm) } & $4.88 \pm 0.71(5)$ & $5.44 \pm 3.82(5)$ & -5.236 & $0.001 * *$ \\
\hline \multicolumn{2}{|l|}{ Postprocedural sPAP (mmHg) } & $38.23 \pm 11.92(37)$ & $40.63 \pm 10.26(38)$ & -3.399 & $0.001 * *$ \\
\hline & & $n(\%)$ & $n(\%)$ & $\chi^{2}$ & p \\
\hline \multirow{2}{*}{ Death } & No & $276(100)$ & $241(96)$ & \multirow{2}{*}{11.209} & \multirow{2}{*}{$0.001 * *$} \\
\hline & Yes & $0(0)$ & $10(4)$ & & \\
\hline \multirow{2}{*}{ Reintervention } & No & $231(83.7)$ & $245(97.6)$ & \multirow{2}{*}{27.545} & \multirow{2}{*}{$0.001^{* *}$} \\
\hline & Yes & $45(16.3)$ & $6(2.4)$ & & \\
\hline \multirow{2}{*}{ Postprocedural functional capacity } & NYHA 1-2 & $261(94.5)$ & $239(95.2)$ & \multirow{2}{*}{0.012} & \multirow{2}{*}{0.910} \\
\hline & NYHA 3-4 & $15(5.5)$ & $16(4.8)$ & & \\
\hline Event-free survival & & $206(81.1)$ & $222(87.9)$ & 2.596 & 0.107 \\
\hline \multirow{2}{*}{ Embolic stroke } & No & $271(98.2)$ & $246(98.0)$ & \multirow{2}{*}{0.023} & \multirow{2}{*}{1.000} \\
\hline & Yes & $5(1.8)$ & $5(2.0)$ & & \\
\hline \multirow{2}{*}{ Endocarditis } & No & 275 (99.6) & 249 (99.2) & \multirow{2}{*}{0.438} & \multirow{2}{*}{0.607} \\
\hline & Yes & $1(0.4)$ & $2(0.8)$ & & \\
\hline \multirow{2}{*}{ Postprocedural rhythm } & SR & $184(66.7)$ & $119(47.4)$ & \multirow{2}{*}{19.166} & \multirow{2}{*}{$0.001^{* *}$} \\
\hline & $\mathrm{AF}$ & $92(33.3)$ & $132(52.6)$ & & \\
\hline
\end{tabular}

${ }^{* *} \mathrm{P}<0.01$; PMBV: percutaneous mitral balloon valvuloplasty; MVR: mitral valve replacement; LA: left atrial; AF: atrial fibrillation; sPAP: systolic pulmonary arterial pressure; NYHA: New York Heart Association; SR: sinus rhythm. 
group had higher MR incidence in our study, corroborating previous studies. In the MVR group, Dhasmana et al. ${ }^{19}$ found a rate of $5.7 \%$ perivalvular leakage; in our study, this rate was 6.4\%. Additionally, like similar studies, we found 2 days hospital stay in the PMBV group, whereas, in the MVR group, hospital stay was 10 days, which is statistically significant.

Cardiac tamponade is one of the serious complications of both PMBV and MVR. In an analysis of 23.000 patients with percutaneous cardiac interventions, cardiac tamponade ratio is highest in PMBV patients ${ }^{20}$. In our study, 4 patients (1.4\%) had a cardiac tamponade due to PMBV, but no deaths were observed in the follow-up.

In our study, mortality rates of PMBV and MVR were $0 \%$ and $4 \%$ respectively, which was statistically significant. Korkmaz et al. ${ }^{11}$ found a $0.7 \%$ death rate on long-term follow-up. Zhang et al. ${ }^{21}$ found that the death rate after MVR was 2-10\% in the early period. In our study, the death rate was $2 \%$ in early stage and $2 \%$ in long-term follow-up. The reason for death was hemorrhagic cerebrovascular disease in three patients, gastrointestinal bleeding in one patient, and unknown in one patient.

The reintervention rate of the PMBV group was $16.3 \%$, and this rate was $2.4 \%$ for the MVR group $(\mathrm{p}<0.001)$. According to previous studies ${ }^{10,22}$, MVR rate after PMBV is $15-27 \%$; in our study, we found it to be $13.8 \%$ (7.9\% on early stage, $5.9 \%$ on long-term follow-up). Karp et al. ${ }^{23}$ found $5 \%$ re-MVR after 5 year follow-up; in our study, $2.4 \%$ of patients needed re-PMBV.

Although PMBV and MVR are accepted gold standard therapy for mitral stenosis, both of them have some important complications. Babic et al. ${ }^{24}$ found a $2 \%$ rate of embolic stroke after PMBV; similarly, in our study, five patients (1.8\%) had embolic stroke. Four of them had embolic stroke on long-term follow-up. On the other hand, Cohen et al. ${ }^{8}$ found $2 \%$ cerebrovascular stroke rate after MVR; similarly, in this study, the cerebrovascular stroke rate was $2 \%$ (five patients) in the MVR group. Infective endocarditis due to mechanical valve is one of the serious complications after MVR, and Katircioglu et al. ${ }^{25}$ have seen it in $2 \%$ of patients on long-term follow-up; in our study, the ratio was $0.8 \%$ on long-term follow-up. Cohen et al. ${ }^{8}$ presents a rate of $13 \%$ of atrial septal defect due to PMBV; in our study, this rate was 3.3\%.

Event-free survival rate of the PMBV group after the follow-up was $81 \%$, and, in the MVR group, it was $88 \%$ ( $\mathrm{p}=0.107$ ).
Song et al. ${ }^{9}$, after 8 years follow-up, found an event-free survival rate of $82 \%$ on PMBV and $86 \%$ on MVR patients. In our study, the $81 \%$ ratio on PMBV and $88 \%$ ratio on MVR patients' event-free survival could explain the increased rate of PMBV treatment for mitral stenosis worldwide, with noninferiority of PMBV to MVR. Our study showed that, although event-free survival of both the PMBV and the MVR groups were similar, the need of reintervention was higher in the PMBV group; contrarily, mortality rate in the MVR group was higher than in the PMBV group.

\section{Study limitations}

The most important limitation of this study was that it was a retrospective study. The other limitation of this study was lack of echocardiographic mitral valve score of patients.

\section{CONCLUSION}

In our study, the event-free survival rates of PMBV and MVR were found to be similar. The need of reintervention was higher in the PMBV group; however, the mortality rate of MVR was higher than PMBV. In conclusion, despite the fact that both modalities are used in the treatment of mitral stenosis, PMBV seems to be more advantageous in selected patients, considering the low mortality rates, easy application of the procedure and no need for general anesthesia.

\section{AUTHORS" CONTRIBUTIONS}

EU: Conceptualization, Data Curation, Formal Analysis, Validation, Writing - Original Draft, Writing - Review \& Editing. RE: Conceptualization, Data Curation, Formal Analysis, Validation. SG: Writing - Original Draft, Writing - Review \& Editing, Validation. AD: Conceptualization, Data Curation, Formal Analysis, Writing - Original Draft, Writing - Review \& Editing. ME: Conceptualization, Data Curation, Formal Analysis. SK: Conceptualization, Data Curation, Formal Analysis, Validation, Writing - Original Draft, Writing - Review \& Editing. MB: Conceptualization, Data Curation, Formal Analysis. NY: Conceptualization, Data Curation, Formal Analysis, Validation, Writing - Original Draft, Writing - Review \& Editing.

\section{REFERENCES}

1. Rahimtoola SH, Durairaj A, Mehra A, Nuno I. Current evaluation and management of patients with mitral stenosis. Circulation. 2002;106(10):1183-8. https://doi.org/10.1161/01. cir.0000029210.14716.01
2. Carapetis JR, Steer AC, Mulholland EK, Weber M. The global burden of group A streptococcal diseases. Lancet Infect Dis. 2005(11):685-94. https://doi.org/10.1016/S14733099(05)70267-X 
3. Carapetis JR, McDonald M, Wilson NJ. Acute rheumatic fever. Lancet. 2005;366(9480):155-68. https://doi.org/10.1016/ S0140-6736(05)66874-2

4. Jose VJ, Gomathi M. Declining prevalence of rheumatic heart disease in rural schoolchildren in India: 2001-2002. Indian Heart J. 2003;55(2):158-60. PMID: 12921331

5. Baumgartner H, Falk V, Bax JJ, De Bonis M, Hamm C, Holm PJ, et al. 2017 ESC/EACTS Guidelines for the management of valvular heart disease. Eur Heart J. 2017;38(36):2739-2791. https://doi.org/10.1093/eurheartj/ehx391

6. Inoue K, Owaki T, Nakamura T, Kitamura F, Miyamoto N. Clinical application of transvenous mitral commissurotomy by a new balloon catheter. J Thorac Cardiovasc Surg. 1984;87(3):394402. PMID: 6700245

7. Martínez-Ríos MA, Tovar S, Luna J, Eid-Lidt G. Percutaneous mitral commissurotomy. Cardiol Rev. 1999;7(2):108-16. https:// doi.org/10.1097/00045415-199903000-00014

8. Cohen JM, Glower DD, Harrison JK, Bashore TM, White WD, Smith LR, et al. Comparison of balloon valvuloplasty with operative treatment for mitral stenosis. Ann Thorac Surg. 1993;56(6):1254-62. https://doi.org/10.1016/00034975(93)90662-2

9. Song JK, Kim MJ, Yun SC, Choo SJ, Song JM, Song H, et al. Long-term outcomes of percutaneous mitral balloon valvuloplasty versus open cardiac surgery. J Thorac Cardiovasc Surg. 2010;139(1):103-10. https://doi.org/10.1016/j. jtcvs.2009.04.022

10. Palacios IF, Sanchez PL, Harrell LC, Weyman AE, Block PC. Which patients benefit from percutaneous mitral balloon valvuloplasty? Prevalvuloplasty and postvalvuloplasty variables that predict long-term outcome. Circulation. 2002;105(12):1465-71. https://doi.org/10.1161/01.cir.0000012143.27196.f4

11. Korkmaz Ş, Demirkan B, Güray Y, Yılmaz MB, Aksu T, Şaşmaz $\mathrm{H}$. Acute and long-term follow-up results of percutaneous mitral balloon valvuloplasty: a single-center study. Anadolu Kardiyol Derg. 2011;11(6):515-20. https://doi.org/10.5152/ akd.2011.136

12. lung B, Garbarz E, Michaud P, Helou S, Farah B, Berdah P, et al. Late results of percutaneous mitral commissurotomy in a series of 1024 patients. Analysis of late clinical deterioration: frequency, anatomic findings, and predictive factors. Circulation. 1999;99(25):3272-8. https://doi.org/10.1161/01.cir.99.25.3272

13. Abascal VM, Wilkins GT, O'Shea JP, Choong CY, Palacios IF, Thomas JD, et al. Prediction of successful outcome in 130 patients undergoing percutaneous balloon mitral valvotomy. Circulation. 1990;82(2):448-56. https://doi.org/10.1161/01. cir.82.2.448

14. Fawzy ME, Shoukri M, Al Buraiki J, Hassan W, El Widaal $H$, Kharabsheh $S$, et al. Seventeen years' clinical and echocardiographic follow up of mitral balloon valvuloplasty in 520 patients, and predictors of long-term outcome. J Heart Valve Dis. 2007;16(5):454-60. PMID: 17944115
15. Herrmann HC, Lima JAC, Feldman T, Chisholm R, Isner J, O'Neill $W$, et al. Mechanisms and outcome of severe mitral regurgitation after Inoue balloon valvuloplasty. North American Inoue Balloon Investigators. J Am Coll Cardiol. 1993;22(3):783-9. https://doi.org/10.1016/0735-1097(93)90191-3

16. Elasfar AA, Elsokkary HF. Predictors of developing significant mitral regurgitation following percutaneous mitral commissurotomy with inoue balloon technique. Cardiol Res Pract. 2011;2011:703515. https://doi. org/10.4061/2011/703515

17. Zaki AM, Kasem HH, Bakhoum S, Mokhtar M, El Nagar W, White CJ, et al. Comparison of early results of percutaneous metallic mitral commissurotome with Inoue balloon technique in patients with high mitral echocardiographic scores. Catheter Cardiovasc Interv. 2002;57(3):312-7. https://doi.org/10.1002/ ccd. 10312

18. Kasem HH, Bakhoum S, Mokhtar M, El-Ramly M, El-Guindy M, Zaki A. Comparison of the immediate results of percutaneous mitral commissurotomy using the metallic valvutome versus Inoue balloon technique. Eur Heart J. 2000;21(Abstract_ Supplement):620. https://doi.org/10.1093/eurheartj/21. Abstract_Supplement.1

19. Dhasmana JP, Blackstone EH, Kirklin JW, Kouchoukos NT. Factors associated with periprosthetic leakage following primary mitral valve replacement: with special consideration of the suture technique. Ann Thorac Surg. 1983;35(2):170-8. https://doi.org/10.1016/s0003-4975(10)61456-7

20. Shen X, Fang Z, Hu X, Liu Q, Zhou T, Tang J, et al. Cardiac perforation and tamponade in percutaneous cardiac intervention. Zhong Nan Da Xue Xue Bao Yi Xue Ban. 2011;36(1):74-9. https://doi.org/10.3969/j.issn.1672-7347.2011.01.012

21. Zhang BR, Xu ZY, Zou LJ, Mei J, Wang ZN, Hao JH. Long-term results of prosthetic mitral valve replacement with home-made tilting disc valve: a report of 125 cases. Zhonghua Wai Ke Za Zhi. 2003;41(4):253-6. PMID: 12882665

22. Hernandez R, Bañuelos C, Alfonso F, Goicolea J, Fernández-Ortiz A, Escaned J, et al. Long-term clinical and echocardiographic follow-up after percutaneous mitral valvuloplasty with the Inoue balloon. Circulation. 1999;99(12):1580-6. https://doi. org/10.1161/01.cir.99.12.1580

23. Karp RB, Cyrus RJ, Blackstone EH, Kirklin JW, Kouchoukos NT, Pacifico AD. The Björk-Shiley valve: intermediate-term follow-up. J Thorac Cardiovasc Surg. 1981;81(4):602-14. PMID: 7206769

24. Babic UU, Grujicic S, Popovic Z, Djurisic Z, Pejcic P, Vucinic M. Percutaneous transarterial balloon dilatation of the mitral valve: five year experience. Br Heart J. 1992;67(2):185-9. https://doi.org/10.1136/hrt.67.2.185

25. Katircioglu F, Yamak B, Battaloglu B, Saritas A, Kiziltepe U, Kural $T$, et al. Long-term results of mitral valve replacement with preservation of the posterior leaflet. J Heart Valve Dis. 1996;5(3):302-6. PMID: 8793680 